\title{
FISIOLOGÍA PULMONAR: La baja eficiencia de la ventilación durante la prueba de ejercicio cardiopulmonar incremental (PECPI), predice mortalidad en pacientes con hipertensión pulmonar (HTP) Low ventilation efficiency during incremental cardiopulmonary exercise test, predicts mortality in patients with pulmonary hypertension
}

Maria José Fernández Sánchez, MD.(1); Javier Iván Lasso Apráez, MD.(2)

Rev Colomb Neumol 2013; 24 (4):223-225

\section{REFERENCIA}

Schwaiblmair M, Faul C, Von Scheidt W, Berghaus TM. Ventilatory efficiency testing as prognostic value in patients with pulmonary hypertension. BMC Pulmonary Medicine. 2012; 12: 23-30.

\section{PREGUNTA}

¿Cuál es el valor predictivo de los parámetros de eficiencia ventilatoria, equivalente ventilatorio (VE/ $\mathrm{VCO}_{2}$ ) y pendiente de $\mathrm{VE} / \mathrm{VCO}_{2}$, para estimar la supervivencia a dos años de pacientes con hipertensión pulmonar arterial (HPA) e hipertensión tromboembólica crónica no operable (HPTEC)?

¿Son Las características operativas de estos parámetros comparables con el consumo pico de oxígeno $\left(\mathrm{VO}_{2}\right.$ pico)?

\section{DISEÑO}

Estudio de cohorte prospectiva.

\section{DURACIÓN}

El período de recolección de pacientes fue de 36 meses.

\section{PACIENTES}

Se incluyeron 116 pacientes. 73 mujeres y 43 hombres. Edad promedio de $63,7 \pm 1,7$ años. Índice de masa corporal de $26,7 \pm 0,4 \mathrm{~kg} / \mathrm{m}^{2}$. Se excluyeron pacientes con enfermedad extra cardiaca severa que limitara la capacidad de ejercicio. 85 pacientes fueron diagnosticados con HPA y 31 con HPTEC. Durante los 24 meses de seguimiento murieron 29 pacientes $(n=21$ en el grupo de HPA y $n=8$ en el grupo de HPTEC). Las causas de muerte fueron falla cardiaca derecha $(n=12)$, falla respiratoria $(n=10)$, hemorragia pulmonar $(n=2)$, arritmia $(n=2)$ y otras causas $(n=3)$.

\section{INTERVENCIÓN}

A todos los pacientes se les hizo espirometría, pletismografía y difusión de monóxido de carbono. Gases arteriales en muestra de sangre capilar arterializada. Caminata de 6 minutos. Prueba cardiopulmonar de ejercicio incremental en bicicleta ergométrica (5-15 W/min) limitada por síntomas de acuerdo con un protocolo estandarizado. Cateterismo cardiaco derecho.

Durante el seguimiento todos los pacientes tuvieron tratamiento farmacológico especifico. Bloqueador de calcio $(7 \%)$, prostanoide inhalado $(13 \%)$, antagonista del receptor de endotelina $(72 \%)$ e inhibidor de fosfodiesterasa-5 $(56 \%)$.

\section{DESENLACE}

Mortalidad por todas las causas.

\footnotetext{
(1) Residente de tercer año de Medicina Interna, Pontificia Universidad Javeriana, Hospital Universitario San Ignacio, Bogotá, Colombia.

(2) Internista, Neumólogo, Hospital Universitario San Ignacio, Pontificia Universidad Javeriana, Bogotá, Colombia.
}

Correspondencia: Dr. Javier Iván Lasso Apráez. Correo electrónico: jilasso.husi@javeriana.edu.co

Recibido: diciembre 10 de 2012. Aceptado: diciembre 20 de 2012. 


\section{SEGUIMIENTO}

El periodo de seguimiento fue de 24 meses. Todos los pacientes completaron el seguimiento.

\section{RESULTADO}

Entre los grupos de pacientes con HTP que sobrevivieron y los que fallecieron se encontraron diferencias significativas en la carga de trabajo lograda $(67,7$ vs. $35,7 \mathrm{~W}, p=0,023)$; en el equivalente respiratorio para oxígeno $\left(\mathrm{VE} / \mathrm{VO}_{2}\right)(42,1$ vs. $56,9, \mathrm{p}=0,001)$; en el $\mathrm{VE} / \mathrm{VCO}_{2}(47,5$ vs. $64,4, \mathrm{p}<0,001)$ y en la pendiente $\mathrm{VE} / \mathrm{VCO}_{2}(47,0$ vs. $65,4, \mathrm{p}=0,014)$. No hubo diferencia significativa en el $\mathrm{VO}_{2}$ pico $(14,4 \pm 1,4 \mathrm{~mL} / \mathrm{min} / \mathrm{kg}$ vs. $10,9 \pm 1,0 \mathrm{~mL} / \mathrm{min} / \mathrm{kg}, \mathrm{p}=\mathrm{NS}$ ) (tabla 1).

Un $\mathrm{VE} / \mathrm{VCO}_{2}$ de 55 o más, mostró un riesgo relativo de mortalidad a 2 años de 7,83 , y sensibilidad de $80 \%$ con especificidad de $79 \%$. Una pendiente de VE/NCO 2 de 60 o más, mostró un riesgo relativo de mortalidad a 2 años de 5,75 , y sensibilidad de $70 \%$ con especificidad de $81 \%$. EI VO $\mathrm{V}_{2}$ pico, con un punto de corte de $10,4 \mathrm{~mL} / \mathrm{kg} / \mathrm{min}$, no fue un buen predictor de mortalidad (riesgo relativo de 1,5 , y sensibilidad de $27 \%$ con especificidad de $69 \%$ ) (tabla 2 ).

\section{CONCLUSIÓN}

Los parámetros de eficiencia ventilatoria, $\mathrm{VE}_{\mathrm{E}} \mathrm{NCO}_{2}$ y pendiente de $\mathrm{VE} / \mathrm{VCO}_{2}$, pronostican confiablemente la evolución a mediano plazo de los pacientes con HPA e HPTEC. No fueron comparables las caracteristicas operativas del $\mathrm{VO}_{2}$ pico como predictor de mortalidad.

\section{COMENTARIO}

La evaluación de pacientes con HTP continúa evolucionando y en los últimos años la PCPEI ha ganado una posición importante como herramienta clínica que proporciona información útil para el diagnóstico y la estratificación de la enfermedad. Ciertos parámetros de la prueba, como el VE/VCO ${ }_{2}$, la pendiente del VE/ $\mathrm{VCO}_{2}$ y el $\mathrm{PetCO}_{2}$ evalúan la desigualdad de la relación ventilación-perfusión, la cual aumenta durante el ejercicio en los pacientes con HTP, dada la incapacidad de reclutamiento del lecho vascular pulmonar remodelado. En consecuencia, la RVP aumenta disminuyendo la perfusión de los alvéolos ventilados, lo cual se traduce en aumento del espacio muerto, así como en disminución del gasto cardíaco (GC) izquierdo y de la entrega tisular de oxigeno $\left(\mathrm{DO}_{2}\right)$.

Tabla 1. Caracteristicas hemodinámicas y cardiopulmonares de los pacientes.

\begin{tabular}{|c|c|c|c|}
\hline & Sobrevivientes $(n=87$ ) & No sobrevivientes $(\mathrm{n}=29$ ) & $\mathbf{p}$ \\
\hline PAP, $\mathrm{mm} \mathrm{Hg}$ & $42,0 \pm 2,5$ & $39,6 \pm 2,6$ & ns \\
\hline $\mathrm{VO}_{2}, \mathrm{~mL} / \mathrm{min} / \mathrm{kg}$ & $14,6 \pm 1,4$ & $10,9 \pm 1,0$ & $\mathrm{~ns}$ \\
\hline W, vatios & $67,7 \pm 8,0$ & $35,7 \pm 1,5$ & 0,023 \\
\hline $\mathrm{VENO} \mathrm{O}_{2}-\mathrm{UA}$ & $42,1 \pm 2,1$ & $56,9 \pm 2,6$ & 0,001 \\
\hline $\mathrm{VENCO}{ }_{2}-\mathrm{UA}$ & $47,5 \pm 2,2$ & $64,4 \pm 2,3$ & $<0,001$ \\
\hline Pendiente $\mathrm{VE} / \mathrm{VCO}_{2}$ & $47,0 \pm 3,2$ & $65,4 \pm 7,5$ & 0,014 \\
\hline a-etCO ${ }_{2}, \mathrm{~mm} \mathrm{Hg}$ & $6,8 \pm 0,8$ & $10,5 \pm 1,1$ & 0,014 \\
\hline $\mathrm{P}(\mathrm{A}-\mathrm{a}) \mathrm{O}_{2}, \mathrm{~mm} \mathrm{Hg}$ & $52,8 \pm 2,8$ & $67,5 \pm-4,2$ & 0,009 \\
\hline
\end{tabular}

PAP: presión arteria pulmonar. W: carga de trabajo. $\mathrm{VO}_{2}$ : consumo pico de oxigeno. VENO $\mathrm{V}_{2}$ equivalente respiratorio de oxigeno. $\mathrm{VENCO}_{2}$ : equivalente respiratorio de dióxido de carbono. a-etCO $\mathrm{CO}_{2}$ diferencia arterio-end-tidal de dióxido de carbono. $\mathrm{P}(\mathrm{A}-\mathrm{a}) \mathrm{O}_{2}$ : diferencia alvéolo arterial de oxigeno. ns: no significativo.

Tabla 2. Parámetros predictores de mortalidad a dos años.

\begin{tabular}{|lcccr|}
\hline & Sensibilidad & Especificidad & $\begin{array}{c}\text { Razón de disparidad } \\
\mathbf{9 5 \%} \text { IC }\end{array}$ & p \\
\hline $\mathrm{VO}_{2}(<10.4 \mathrm{~mL} / \mathrm{min} / \mathrm{kg})$ & $27 \%$ & $69 \%$ & $1,88[1,58 ; 2,18]$ & 0,03 \\
$\mathrm{VE} / \mathrm{VCO}_{2}(\geq 55)$ & $80 \%$ & $79 \%$ & $14,66[12,70 ; 16,62]$ & 0,004 \\
$\mathrm{VEN} \mathrm{VCO}_{2}(\geq 60)$ & $70 \%$ & $81 \%$ & $9,92[6,68 ; 13,17]$ & 0,03 \\
\hline
\end{tabular}

$\mathrm{VO}_{2}$ : consumo pico de oxigeno. $\mathrm{VENCO}$ : equivalente respiratorio de dióxido de carbono. $\mathrm{P}(\mathrm{A}-\mathrm{a}) \mathrm{O}_{2}$; diferencia alvéolo arterial de oxígeno. a-et $\mathrm{CO}_{2}$ : diferencia arterio-end-tidal de dióxido de carbono. 
D’Alonzo evaluó en 1987 la respuesta ventilatoria y el intercambio gaseoso durante el ejercicio en pacientes con HTP primaria (1), y halló un aumento progresivo de la pendiente de $\mathrm{VE} / \mathrm{NCO}_{2}$ y de la $\mathrm{P}(\mathrm{A}-\mathrm{a})$ $\mathrm{O}_{2}$. Yasunobu describió una disminución del $\mathrm{PetCO}_{2}$ dependiente de la severidad en pacientes con HTP primaria tanto en reposo como en ejercicio (2).

Con la progresión de la hipertensión es frecuente que se permeabilice el foramen oval y se produzca un corto-circuito de derecha a izquierda que se manifiesta con un aumento abrupto del $\mathrm{VE} / \mathrm{NCO}_{2}$ y una disminución inclinada del $\mathrm{PetCO}_{2}(3)$.

Otro parámetro de la PCPEI que refleja la severidad de la enfermedad es el $\mathrm{VO}_{2}$ pico (4), el cual estará disminuido como resultado de la caída del GC, que se explica por un volumen bajo de llenado del ventrículo izquierdo y por el movimiento paradójico del septum interventricular (5). La acidosis láctica temprana resultante de la hipoperfusión muscular durante el ejercicio, estimula la VE dado que hay un aumento en la producción de $\mathrm{CO}_{2}$ como consecuencia del taponamiento de ácido láctico con bicarbonato, lo cual se ve reflejado en el aumento del $\mathrm{VE} / \mathrm{VCO}_{2}(6)$.

Conociendo el comportamiento de estos parámetros de ejercicio en pacientes con HTP, Schwaiblmair en su trabajo confirmó el valor del $\mathrm{VE} / \mathrm{VCO}_{2}$ como mejor predictor de mortalidad a mediano plazo en pacientes con HPA e HPTEC no operable con tratamiento farmacológico específico. Contrariamente, el $\mathrm{VO}_{2}$ pico $(\mathrm{mL} / \mathrm{kg} / \mathrm{min})$ que ha sido un parámetro usado por mu- cho tiempo, no tuvo unas características operativas satisfactorias como predictor de muerte.

Con lo anterior se puede decir que se consolida cada vez más la utilidad de la PCPEI en la evaluación integral de los pacientes con HTP, y se establece como un método no invasivo relativamente fácil y reproducible que debe emplearse con mayor frecuencia en el proceso de diagnóstico y estratificación de riesgo de la enfermedad vascular pulmonar.

\section{BIBLIOGRAFIA}

1. D'Alonzo GE, Gianotti LA, Pohil RL et al. Comparison of progressive exercise performance of normal subjects and patients with primary puimonary hypertension. Chest 1987; 92: 57-62.

2. Yasunobu $Y$, Oudiz RJ, Sun XG, Hansen JE, Wasserman $K$. End-tidal PCO2 abnormality and exercise limitation in patients with primary pulmonary hypertension. Chest 2005; 127: 16371646.

3. Sun XG, Hansen JE, Oudiz RJ, Wasserman K. Gas exchange detection of exercise-induced right-to-left shunt in patients with primary pulmonary hypertension. Circulation 2002; 105: 54-60.

4. Arena R, Lavie CJ, Milani RV, Myers J, Guazzi M. Cardiopulmonary exercise testing in patients with pulmonary arterial hypertension: an evidence-based review. J Heart Lung Transplant. 2010; 29: 159-173.

5. Tji-Joong Gan C, Lankhaar JW, Marcus JT et al. Impaired left ventricular filling due to right-to-left ventricular interaction in patients with pulmonary arterial hypertension. Am J Physiol Heart Circ Physiol. 2006; 290: 1528-1533.

6. Sun XG, Hansen JE, Oudiz RJ, Wasserman K. Exercise pathophysiology in patients with primary pulmonary hypertension. Circulation 2001; 104: 429-435. 